Artículo de investigación.

Cómo citar:

Editorial: Corporación Universitaria Minuto de Dios - UNIMINUTO.

Recibido: 30 de enero de 2018 Aceptado: 2 de abril de 2018 Publicado: 1 de agosto de 2018

Conflicto de intereses: los autores han declarado que no existen intereses en competencia.
Elvis Antonio Carrero Sepúlveda elviscarrero@yahoo.com

Magister en Desarrollo Sostenible y Medio Ambiente de la Universidad de Manizales. Profesional en Relaciones Internacionales y Estudios Políticos.

Óscar Fernando Gómez Morales oscar@umanizales.edu.co Docente investigador en el Centro de Investigaciones en Medio Ambiente y Desarrollo (Cimad) de la Universidad de Manizales.

\section{Desarrollo social y humano y su aporte a la paz en Turbaco (Bolívar, Colombia)}

\author{
Social and Human Development: Their \\ Contribution to Peace in Turbaco \\ (Bolívar, Colombia)
}

\section{Desenvolvimento social e humano e seu contribua à paz em Turbaco (Bolívar, Colômbia)}

Elvis Antonio Carrero Sepúlveda, Óscar Fernando Gómez Morales

\section{Resumen}

Este artículo de investigación interpreta la contribución al desarrollo sostenible y a la paz en el municipio de Turbaco (Bolívar, Colombia), a través de programas desarrollados por parte de una empresa privada. El análisis de estos programas, en compañía de los líderes de los desplazados, señala aquellos programas e iniciativas aportantes al desarrollo sostenible y a la paz y abre un abanico de posibilidades para que el sector empresarial público y privado siga nuevos modelos que contribuyan a la paz y al desarrollo social en aquellas comunidades donde tienen presencia.

Se revisó un periodo entre 2014 y 2016, incluyendo 107 encuestas con miembros de la comunidad desplazada y entrevista a la directora de la empresa privada que implementó los programas.

Palabras clave: desarrollo sostenible, paz, sostenibilidad y conflicto armado colombiano.

\begin{abstract}
This research paper examines the contribution to sustainable development and peace in the municipality of Turbaco (Bolívar, Colombia), of programs implemented by a private company. The analysis, supported by leaders of the displaced people, shows those programs and initiatives which contribute to sustainable development and peace, and opens up a variety of possibilities so that the public and private companies follow new models that favor peace and social development in the communities where they are located.
\end{abstract}


The study focuses in the 2014-2016 term. It includes 107 surveys administered to members of the displaced community and an interview to the manager of the private company responsible for the programs.

Key words: sustainable development, peace, sustainability and armed conflict in Colombia.

\section{Resumo}

Este artigo de investigação interpreta a contribuição ao desenvolvimento sustentável e à paz no município de Turbaco (Bolívar, Colômbia), através de programas de desenvolvimento por parte de uma empresa privada. A análise destes programas, em companhia dos líderes dos deslocados, assinala aqueles programas e iniciativas aportantes ao desenvolvimento sustentável e à paz e abre uma gama de possibilidades para que o setor empresarial público e privado siga novos modelos que contribuam à paz e ao desenvolvimento social naquelas comunidades onde têm presença. Revisou-se um período entre 2014 e 2016, incluindo 107 questionários com membros da comunidade deslocada e entrevista à diretora da empresa privada que implementou os programas.

Palavras-chave: desenvolvimento sustentável, paz, sustentabilidade e conflito armado colombiano.

\section{Introducción}

La naturaleza del conflicto armado colombiano conlleva una serie de problemáticas que han dificultado los acuerdos de paz entre el Estado colombiano y los grupos armados ilegales. Esto a su vez ha estimulado a que la sociedad colombiana, sectores de la industria, de la academia, de las ONG, organismos internacionales, busquen alternativas que redunden en avances para lograr una paz que minimice los alcances del conflicto. En una sociedad imperfecta, que manifiesta síntomas de inequidad social, pobreza, violencia y contaminación del medio ambiente, se han volcado, como se dice más arriba, diferentes entidades públicas y privadas, que contribuyen al desarrollo del país, con el fin de emprender nuevos caminos que contribuyan a la tarea permanente de reconfiguración y sostenibilidad de la sociedad colombiana.

Esa búsqueda de la consecución de la paz y mejorar la calidad de vida de las poblaciones afectadas ha suscitado que organismos internacionales y el sector privado se unan, con el fin de contribuir a dichos propósitos que involucren a toda la sociedad colombiana.

Una manera específica de contribuir con estos propósitos por parte del sector empresarial colombiano ha sido la adhesión de varias industrias y empresas a la iniciativa Negocios para la Paz. Esta plataforma lanzada por el Pacto Global de las Naciones Unidas en septiembre de 2013 busca contribuir desde su proceso de negocio a encontrar acciones que sean y vayan encaminadas en el avance de la paz en zonas de su influencia y que representan un alto riesgo en el conflicto. 
En palabras de Melissa Powell, directora de Estrategias y Alianzas de la onU: "Negocios para la Paz está basado en diez principios que tienen que ver básicamente con la Declaración Universal de los Derechos Humanos, trabajo, medio ambiente, anticorrupción y otros que han acordado los gobiernos internacionales sobre convenciones" (Paz, 2017).

Este artículo presenta avances del proceso de investigación, donde se identifican nuevos mecanismos y planes encaminados al avance de la paz y el desarrollo social en la comunidad desplazada y en los habitantes del municipio de Turbaco, situado a $10^{\circ} 19^{\prime}$ 30 " de latitud norte y a $1^{\circ} 17^{\prime} 29^{\prime \prime}$ de longitud oeste del meridiano de Bogotá y ubicado a 10 kilómetros de Cartagena de Indias (Bolívar, Colombia). Estos aportes llegan a ser importantes en una región del Caribe colombiano y pueden ser significativos para minimizar las causas del conflicto. El objeto de la investigación se centra en el municipio de Turbaco, afectado por amenazas a la población por actores armados ilegales o grupos de delincuencia, que han venido de otras regiones del país en condición de desplazados y que se han asentado en este municipio. Se establece como objetivo general interpretar la contribución al desarrollo sostenible y a la paz en el municipio de Turbaco (Bolívar, Colombia), a través de programas desarrollados por parte de un sector empresarial privado en esta región del país. Para ello se analizaron las afectaciones sociales y a la paz generadas a partir del desarrollo de los programas implementados por el sector privado e identificar nuevos mecanismos y planes que vayan encaminados al avance de la construcción de la paz y el desarrollo social en la comunidad desplazada.

\section{Antecedentes del estudio}

La Comisión Mundial para el Medio Ambiente y Desarrollo de las Naciones Unidas, a partir del conocido Informe Brundtland (1987), en su reporte a la Asamblea General de la ONU, definió el desarrollo sostenible como "[...] aquel desarrollo que satisface las necesidades del presente sin comprometer la capacidad de las generaciones futuras de satisfacer las propias". Esta definición demuestra la responsabilidad que tienen las actuales generaciones frente a las que vendrán en el futuro. Ello propone un reto grande a las generaciones presentes, debido a que se deben buscar los mecanismos y medios, con el fin de que las futuras generaciones puedan gozar de los mismos beneficios que tenemos hoy en día, de lo contrario se pondrá en peligro la subsistencia de dichas generaciones. Pearce (1988, citado por Bifani, 1997, p.115) ha definido el desarrollo sostenible en "[...] ser justo o equitativo con el futuro", lo que ratifica el compromiso que tienen las personas con quienes vendrán en futuro cercano. Vale la pena resaltar que las generaciones futuras no solo heredarán un ambiente más contaminado, con menos recursos naturales para su subsistencia y recursos renovables escasos. Se debe tener presente que pueden llegar a heredar estilos de cultura diferentes que afectan a la sociedad, modelos estatales en caos, regímenes que contradicen modelos sostenibles, crisis políticas y países con guerras internas que conducen a violaciones de derechos humanos y minimizan la calidad de vida de las personas. El desarrollo sostenible va estrechamente ligado a todos los aspectos de la vida humana, no solamente vinculado con el crecimiento económico, como se tiende a creer. Para Bifani (1997), en su libro Medio ambiente y desarrollo, hace una aclaración al concepto de desarrollo, indicando que este debe ser visto desde una 
perspectiva multidimensional, que incluye elementos económicos, políticos y sociales, así como aquellos relacionados con el uso de los recursos e impactos del medio ambiente.

Lo anterior lo corrobora y aclara un artículo publicado por la Organización de las Naciones Unidas para la Educación, la Ciencia y Cultura (2012): "Educación para el Desarrollo Sostenible Libro de Consulta” (2017), donde indica que todos los programas para el desarrollo sostenible deben considerar tres ámbitos de la sostenibilidad: medio ambiente, sociedad y economía; así como una dimensión subyacente de la cultura. Resalta ideales y principios que constituyen la sostenibilidad, incluyendo conceptos amplios como equidad entre generaciones, equidad de género, paz, tolerancia, reducción de la pobreza, preservación y restauración del medio ambiente, conservación de los recursos naturales y justicia social.

Lo anterior demuestra que el desarrollo sostenible conlleva inmersos estos principios y que, por ende, son y están interrelacionados para que se logren las condiciones de sostenibilidad por los que propenden hoy día muchos países y sociedades.

La conferencia de las Naciones Unidas sobre el Medio Ambiente y el Desarrollo o Cumbre de la Tierra (Río de Janeiro, 1992) dio como resultado la Declaración de Río, estipulando 27 principios, de los que se resaltan cuatro:

1. Los seres humanos tienen derecho a una vida saludable y productiva en armonía con la naturaleza.

2. Erradicar la pobreza y reducir las disparidades en los niveles de vida en los distintos pueblos del mundo es indispensable para el desarrollo sostenible.

3. La guerra es intrínsecamente destructiva para el desarrollo sostenible. La paz, el desarrollo y la protección del medio ambiente son interdependientes e inseparables.

4. La guerra es, por definición, enemiga del desarrollo sostenible. En consecuencia, los Estados deberán respetar las disposiciones de derecho internacional que protegen al medio ambiente en épocas de conflicto armado y cooperar en su ulterior desarrollo, según sea necesario.

Estos principios demuestran las razones por las que la paz hace parte del desarrollo de una región, pueblo o país, señalando que forma parte de la sostenibilidad que deben alcanzar las diversas comunidades de la sociedad.

La paz está intrínsecamente relacionada con el desarrollo sostenible y hablar de esta permite adentrarse en algunas reflexiones hechas por diferentes autores y conocer esa relación entre la paz, el desarrollo humano y la sostenibilidad. La paz busca equilibrio entre las relaciones existentes en los seres humanos, generando un estado de armonía e interrelación colectiva. Como mencionan Muñoz et al. (2005) en su libro Investigación de la paz y los derechos humanos desde Andalucia, la paz es vista como: 
Aquella que representa a todas las acciones humanas encaminadas a preservar el más alto grado de bienestar de las personas, los grupos y la especie. La paz es un signo de bienestar, felicidad y armonía que nos une a los demás, también a la naturaleza y al universo en su conjunto. La paz nos hace sentirnos más humanos y les da sentido a nuestras vidas. Nos facilita relacionarnos los unos con los otros como miembros de una misma especie independientemente de las diferencias que por una u otra razón puedan existir entre nosotros. (pp.13-14).

Este concepto demuestra que la sociedad, en su afán de progreso, debe mantener entre sus ciudadanos relaciones idóneas, que permitan el surgimiento de esta, desde esferas políticas, económicas, religiosas, culturales, étnicas y sociales. Se debe reconocer la paz como aquel elemento constitutivo para el desarrollo de una sociedad que permitirá ser una guía en las acciones que llegaren a tomar los seres humanos.

La paz tiende a desaparecer cuando irrumpe, en todos y en cada uno de nosotros, el pensamiento de guerra, cuando convertimos a los otros en depositarios exclusivos del mal (Cardona et al., 2004, p. 29). Desde esta premisa es factible que el dinamismo de la paz en la sociedad sea tan frágil que lleve a estados de violencia y guerra entre el conglomerado y entre aquellos países depositarios de una soberanía nacional. Cuando se rompe un legado de paz en una sociedad, se debe empezar por edificar nuevamente dicho legado. Esta construcción de paz consiste en acciones dirigidas a identificar y apoyar estructuras tendientes a fortalecer y solidificar la paz para evitar una recaída al conflicto (ONU, 1992).

Entender hoy lo que ocurre, con los desplazados que habitan en el municipio de Turbaco y los posibles mecanismos de construcción de paz ante un eventual posconflicto en Colombia, lleva a revisar un artículo realizado por Angelika Rettberg (2003), quien describe e identifica el tema de la construcción de la paz para el posconflicto. Para el caso colombiano existe una gran correlación, debido a que dicha construcción plantea una agenda que propone retos y metas, e involucra a actores nacionales e internacionales, interesados en hacer aportes significativos en la construcción de la paz en Colombia.

Por ello determinar en qué momento se rompió ese legado de paz en Colombia suscita la reflexión sobre las causas y orígenes que motivaron a que se desarrollase un conflicto en el país. Kurtenbach y Romero (2005), en su libro Análisis del conflicto en Colombia, plasman claramente las causas estructurales del conflicto e identifican a los actores que forman parte de este. Cuatro conjuntos de causas son el eje central del conflicto: Una cultura política de violencia, la debilidad del Estado colombiano, los límites de la participación política y el acceso desigual a la tierra y los recursos naturales. No podemos dejar de lado las principales manifestaciones violentas del conflicto, como los son el secuestro, el desplazamiento forzado, las masacres, los homicidios, los enfrentamientos directos y los ataques a la infraestructura del Estado. Estas manifestaciones son las que ocasionan una maximización del conflicto en el país y el éxodo masivo de campesinos hacia lugares menos hostiles como ciudades o municipios, donde los índices de violencia sean más bajos.

Este desplazamiento masivo es resultado de la violación a los derechos humanos, lo que trasciende en el desarrollo sostenible de una región o municipio. Un ejemplo del desplazamiento masivo lo han vivido varios municipios del departamento de Bolívar, en especial El Carmen de Bolívar, perteneciente a los Montes de María, con altos índices de 
población desplazada hacia otros municipios del mismo departamento, como Cartagena de Indias (capital de Bolívar) y Turbaco (cercano a Cartagena).

\section{Descripción metodológica}

El desarrollo metodológico se ha realizado bajo una aproximación hermenéutica, bajo el orden cualitativo, pretendiendo observar el contexto social y humano de la comunidad objeto de estudio, en este caso, el del municipio de Turbaco. La investigación se realizó durante 18 meses entre 2014 y 2016, incluyendo 107 encuestas con preguntas semiestructuradas (cerradas y abiertas) a miembros de una comunidad de desplazados habitantes de los barrios El Talón y Bonanza.

Con esta metodología se buscó describir y analizar la realidad encontrada, desde el punto de vista de las comunidades desplazadas. Se usó la técnica de grupo focal, con la participación de líderes de los desplazados y sus historias de vida, con el fin de conocer por parte de estos la percepción del aporte social y a la paz que puede hacer una empresa de carácter privado en sus comunidades.

Se analizaron varios documentos (tabla 1), referenciados en el concepto del desarrollo sostenible y aquellos textos que permitieron tener una mejor comprensión del conflicto armado colombiano y su relación con el primer concepto enunciado.

Tabla 1. Documentos revisados sobre el concepto de desarrollo sostenible

\begin{tabular}{|l|l|c|}
\hline \multicolumn{1}{|c|}{ AUTOR } & \multicolumn{1}{|c|}{ LIBRO } & FECHA \\
\hline Pablo Bifani & Medio ambiente y desarrollo & 1997 \\
\hline $\begin{array}{l}\text { Manfred Max-Neef, Anto- } \\
\text { nio Elizalde, y MartinHo- } \\
\text { penhay }\end{array}$ & $\begin{array}{l}\text { Desarrollo a escala humana: } \\
\text { Conceptos, aplicaciones y } \\
\text { algunas reflexiones }\end{array}$ & 2094 \\
\hline Germán Rey & $\begin{array}{l}\text { Cultura y desarrollo huma- } \\
\text { no: unas relaciones que se } \\
\text { trasladan }\end{array}$ & 2002 \\
\hline
\end{tabular}

Fuente: Elaboración propia (2016).

La revisión del Informe de Sostenibilidad (2014), emitido por Surtigas S.A. ESP, empresa dedicada a la comercialización de gas natural en la Costa Caribe, en adelante denominada empresa privada, y la indagación de los diversos programas de responsabilidad empresarial implementados hasta ahora, permitieron conocer su orientación y postura frente al desarrollo sostenible y a la paz.

Esta investigación, siendo de carácter cualitativo, hace una aproximación a las ciencias humanas, permitiendo examinar, explicar, describir, interpretar y analizar los hechos observados a través de las técnicas utilizadas y los cuestionamientos que se formularon a la directora de la Fundación Surtigas, a los líderes y a las personas de la comunidad desplazada. 


\section{Resultados}

Las encuestas aplicadas han permitido conocer las percepciones de los encuestados respecto de los programas implementados por un sector empresarial privado, teniendo en cuenta como ejemplo a esta empresa y su posible contribución a la paz y al desarrollo sostenible y asentado esta información acorde a los objetivos de la presente investigación.

Un primer interrogante realizado a los desplazados acerca de lo que han escuchado sobre esta empresa privada y su fundación da como primer resultado (figura 1) que el $57 \%$ de las personas saben que la organización ayuda a las personas a capacitarse en proyectos productivos y en iniciación de negocios. Este último aspecto se ha orientado en especial a madres cabeza de hogar. El restante $43 \%$ de los encuestados afirman no conocer la fundación de la empresa y el rol que desarrolla.

Figura 1. ¿Conoce usted o ha escuchado sobre la Fundación Surtigas y los proyectos implementados? ¿Cuáles proyectos?

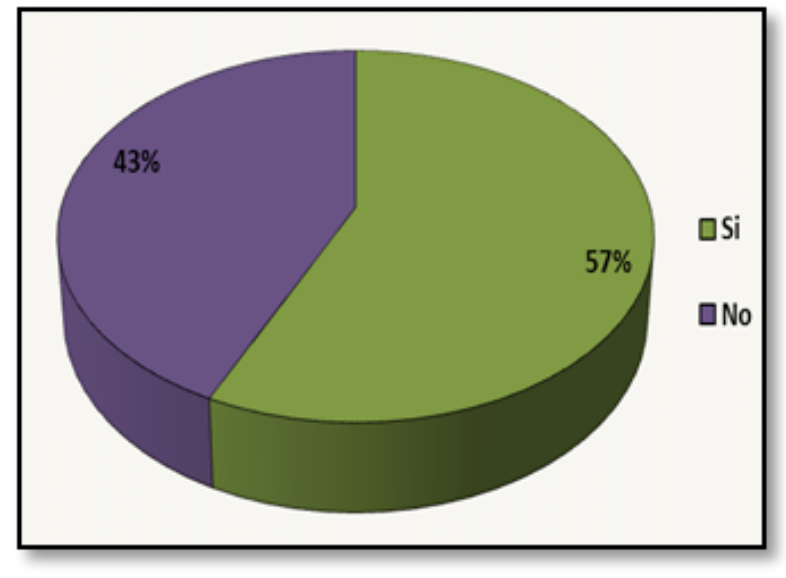

Fuente: Elaboración propia (2016).

Otro de los resultados significativos establece que un $48 \%$ de las personas encuestadas mencionan un programa bandera desarrollado por la organización, programa que busca capacitar a los jóvenes mayores de 18 años como técnicos en instalaciones de gas natural, logrando con ello generar empleabilidad entre la población desplazada.

La gran mayoría de las personas desplazadas consideran que los programas implementados por parte de la empresa privada contribuyen a la paz y al desarrollo social, ya que estos ayudan a las comunidades a mejorar su calidad de vida, permitiendo a las personas de escasos recursos obtener una formación y educación logrando, de esta manera, la inserción a una vida laboral y el mejoramiento de su condición y superación de la pobreza.

Los encuestados comparten, como un gran común denominador, que al realizarse nuevos programas educativos, capacitaciones a la juventud y en general a la comunidad en temas como sexualidad, prevención de drogas, efectos del consumo de alcohol y formación a la comunidad sobre valores en la familia, contribuirían aún más al forjamiento de la paz en la comunidad desplazada que habita en el municipio. 
El $97 \%$ de las personas encuestadas (figura 2) consideran que el sector empresarial privado, desde su actuar de responsabilidad corporativa, puede desarrollar e implementar nuevos mecanismos que contribuyan a la paz en la comunidad turbaquera y demás municipios del país; teniéndose como ejemplo los programas sociales que ha desarrollado la empresa privada en cuestión en los municipios del departamento de Bolívar y que han ayudado al forjamiento de la paz en esas comunidades.

Figura 2. ¿Cree usted que la empresa puede hacer un aporte que contribuya a la paz en la comunidad turbaquera? ¿Por qué?

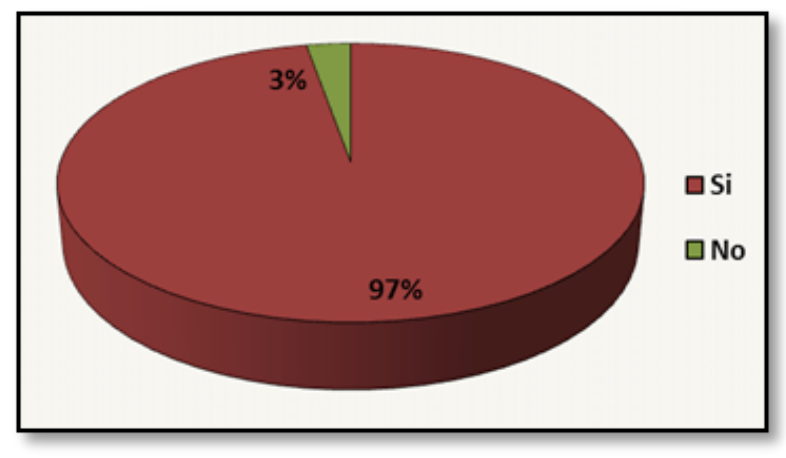

Fuente: Elaboración propia (2016).

Para las víctimas del desplazamiento, se evidencia (figura 3) la existencia de una serie de factores como inseguridad en la región, desempleo, violencia intrafamiliar, desmotivación y falta de credibilidad por parte de la comunidad hacia nuevos programas, analfabetismo, falta de respaldo de entes municipales y desactualización del registro de víctimas desplazadas por parte de la alcaldía; factores determinantes que impiden la ejecución de nuevos programas enfocados a la paz y el desarrollo social en la comunidad. 
Figura 3. Factores que pueden impedir la ejecución de nuevos programas enfocados a la paz y el desarrollo social. ¿Por qué?

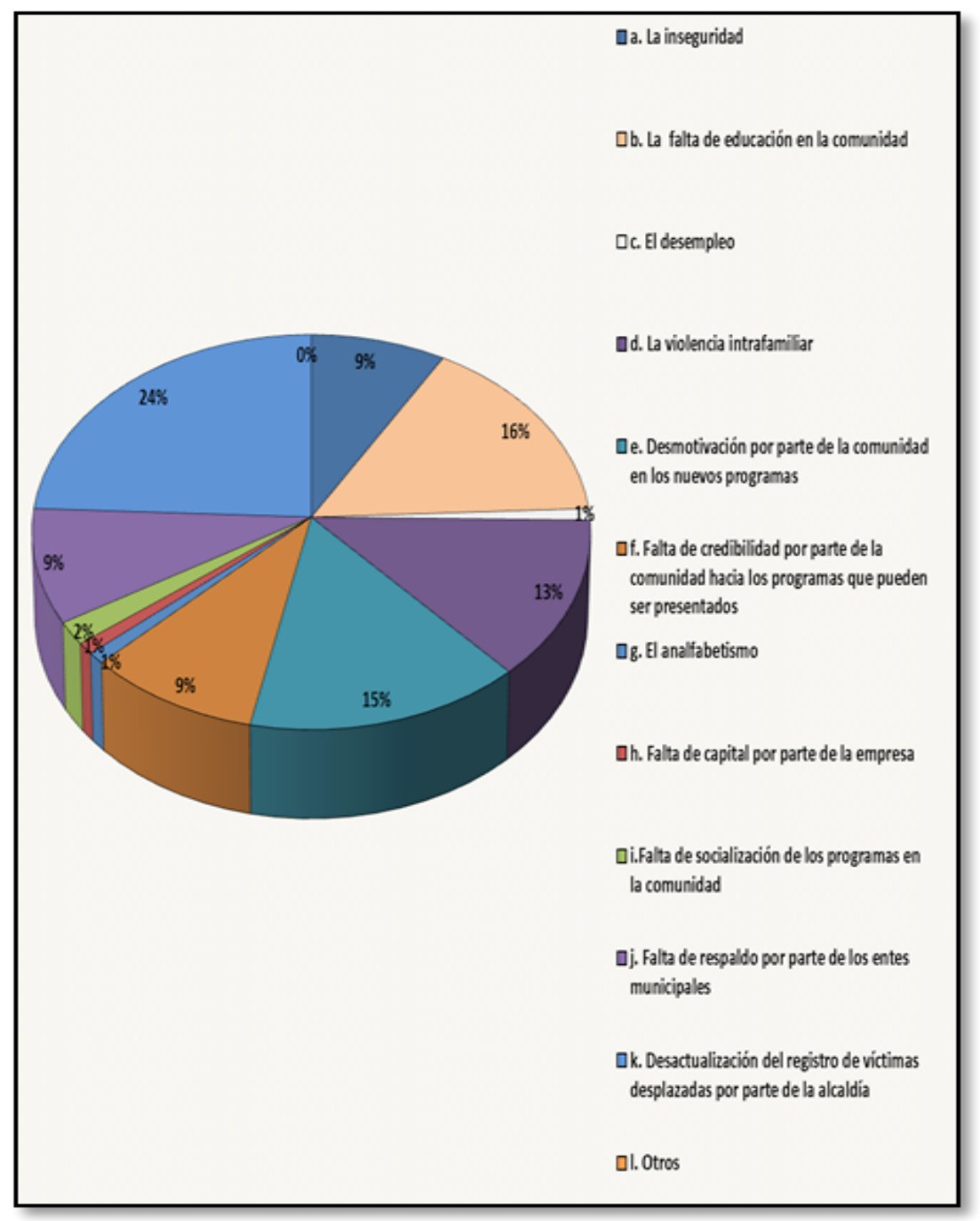

Fuente: Elaboración propia (2016).

Estos resultados muestran que, hoy en día, el sector empresarial privado es concebido como agente de cambio social en aquellas comunidades desplazadas, asumiendo un compromiso hacia estas, ya que inciden en la construcción de la paz, en poblaciones ubicadas en regiones víctimas del conflicto armado colombiano. Sus aportes al desarrollo social van más allá de aquellos territorios donde están presentes; ejemplo de ello es visto por los desplazados de Turbaco a través de esta empresa privada de la región, lo que ha permitido tener un reconocimiento como miembros de la sociedad y no solo como agentes de mercado, facilitándoles la participación en escenarios de transformación social donde, finalmente, la organización logra dinamizar procesos en las comunidades a largo plazo, apoyando las políticas públicas, gestionando, con visión de sostenibilidad, temas sociales, ambientales y financieros. 
La adhesión a la iniciativa Negocios por la Paz, por parte de empresas privadas llevada a cabo hace cuatro años responde a prácticas de responsabilidad corporativa que permiten y facilitan la construcción de paz en territorios que han sufrido las consecuencias del conflicto colombiano. Un ejemplo es el aporte realizado por empresas privadas que, a través de fundaciones sin ánimo de lucro, implementan programas con jóvenes desplazados, permitiéndoles obtener oportunidades de empleabilidad e inserción laboral (oEI, según Rey, 2002).

Tal ha sido el caso de la Fundación Surtigas, donde su práctica de responsabilidad social es focalizada en los siguientes tres aspectos:

1. La prevención, donde trabajan con aquellos jóvenes en riesgo social, capacitándoles en temas como ciudadanía y promoción de sus derechos humanos.

2. Acciones de reparación, a través de programas sociales dirigidos a víctimas desplazadas por la violencia, creándoles oportunidades de emprendimiento, inserción laboral o siendo partícipes de un negocio inclusivo.

3. Reincorporación a la vida civil, mediante realización de trabajos pilotos con personas reinsertadas de la violencia.

El programa que le ha aportado al desarrollo sostenible y la paz en el municipio ha sido Jóvenes con valores productivos, donde han incluido a la población en condición de desplazamiento. Este programa permitió que mujeres desplazadas se formaran como técnicos en instalación de gas natural, logrando ser incluidas en la cadena de valor de la empresa.

\section{Lo que opina el grupo focal}

Los resultados del grupo focal permitieron conocer que la empresa es conocida por la comunidad como una entidad de servicio público y también como una organización que, a través de su fundación, ha beneficiado a un grupo de desplazados mediante programas sociales, permitiéndoles obtener una formación en diversas temáticas; visto lo anterior como aporte a la paz, tal y como lo manifiesta una líder en el siguiente aparte: “... la persona formada y con opciones de empleabilidad se forja en un mejor ciudadano, quien logra romper el ciclo de inseguridad, pobreza y violencia que traen consigo cuando sufre el desplazamiento forzado" (Relato de líder de los desplazados, 2016).

El desarrollo económico y social en este tipo de comunidades trae consigo bienestar común generando una sociedad productiva con diversas capacidades, que finalmente otorga prosperidad en estas.

\section{Historias de vida}

En consonancia con los objetivos del presente instrumento investigativo, se presentan las historias de vida que compartieron algunas personas. Se identifica que el sector privado, de alguna forma, ha ayudado a aquellas comunidades de desplazados en los barrios El Talón y Bonanza, permitiéndoles mejorar su condición de vida, al recibir una 
capacitación con el fin de implementar un negocio propio u obtener formación, para luego ser vinculados a la misma empresa que ha vinculado a jóvenes capacitados como técnicos instaladores de gas natural.

A continuación se presenta un fragmento de una impresión sobre la experiencia vivida por una madre:

Yo participé de un programa que promocionó la empresa, donde se me capacitó para crear un negocio independiente. Me enseñaron sobre la manera de administrar el dinero y lo relacionado con el mercadeo para establecer mi propio negocio. (Relato de una mujer cabeza de hogar, 2016).

\section{Análisis documental}

Las memorias de sostenibilidad muestran el modelo de responsabilidad social empresarial que tienen implementado las empresas, frente a sus grupos de interés, en especial con aquellas comunidades donde buscan una alianza para ser un actor de incidencia en las dinámicas sociales, históricas y culturales de esas comunidades, reafirmando el concepto de empresa familiarmente responsable que escucha, dialoga y promueve valores ciudadanos.

La iniciativa de adhesión al CoP (Comunicación del Progreso) del Pacto Global de Naciones Unidas, desde la Red Pacto Global Colombia (2011), por parte de una empresa privada de la Costa Caribe, es una muestra de compromiso que se adquiere frente a la construcción del desarrollo social, los 10 principios que promueve el pacto en las dimensiones de derechos humanos, estándares laborales, medio ambiente y lucha contra la corrupción.

De igual forma, se expresa la voluntad de continuar participando en el aprendizaje, el diálogo y la acción colectiva de promover la paz en el lugar de trabajo, en los mercados y en las comunidades locales, adoptando las recomendaciones de la guía de lineamientos sobre responsabilidad empresarial en áreas de alto riesgo y afectadas por conflictos, contenidas en la iniciativa empresarial Negocios para la Paz.

\section{Programa Brilla de Surtigas: Modelo de negocios inclusivos}

El programa Brilla ${ }^{1}$ de Surtigas: Modelo de negocio inclusivo, que funciona como sistema de crédito preaprobado para las familias más activas y fieles al servicio de gas natural. Este esquema de financiación no bancaria facilita a los usuarios de estratos más bajos acceso a cupo de crédito para compra de productos como materiales de construcción, equipos, herramientas, artículos para el hogar, computadores, útiles escolares y pago de matrículas universitarias.

1 Brilla obtuvo la distinción "Inclusive Business Leader Award 2011", de International Finance Corporation (IFC) -Banco Mundial, que lo define como modelo de negocio inclusivo que aporta a la mitigación de la pobreza del país (Informe de Sostenibilidad Surtigas, 2013. p. 32). 
Gracias al impacto social en Turbaco y en otros municipios de Bolívar, Sucre y Córdoba, el programa se ha posicionado como un esquema innovador, beneficiando a muchas familias de escasos recursos para mejorar sus condiciones de vida.

\section{El programa Jóvenes con Valores Productivos}

El programa Jóvenes con Valores Productivos, es fruto del diálogo con contratistas, colaboradores y la comunidad, donde se identificó la oportunidad de formar como técnicos en gas natural a jóvenes de Cartagena, Montes de María y Córdoba, que se encontraban en situación de desplazamiento y vulnerabilidad, para luego insertarlos laboralmente a la organización y a su red de empresas contratistas.

Los ejes temáticos desarrollados, como fortalecimiento a organizaciones de base, inserción laboral, emprendimientos y gestores sociales, dieron la oportunidad a jóvenes de escasos recursos para que accedieran a un crédito por la vía de un fondo local para el establecimiento o fortalecimiento de un negocio, bien fuese comercio de mercancía, prestación de un servicio o manufactura.

Mejorar la calidad de vida significó en algunos jóvenes la posibilidad de continuar con otros estudios técnicos, tener una independencia económica de sus padres y, a su vez, logró la equidad de género, al capacitar a mujeres que demostraron tener las mismas o mejores capacidades para desempeñar este trabajo, que no era exclusivo para desarrollar por parte de los hombres.

\section{Resultado de la investigación frente a otros estudios de desarrollo social y la paz}

A lo largo del proceso investigativo, se ha encontrado que los lineamientos adoptados por una empresa del sector privado de la Costa Caribe colombiana, frente a un desarrollo social en las comunidades, establecen la importancia que tiene una organización con sus grupos de interés, buscando tener presente no solo las expectativas, sino las necesidades de los grupos poblacionales de desplazados.

Lo anterior ha llevado a la búsqueda de mecanismos idóneos, a través de programas y proyectos, con el fin de establecer un modelo de sostenibilidad que lleva inmersa la paz, contribuyendo, no de manera asistencialista, sino a través de un desarrollo que genere estabilidad y prosperidad bajo un enfoque participativo por parte de la comunidad y entes gubernamentales, logrando con ello la construcción de comunidades sostenibles.

Un artículo de Villar (2015), denominando Comunidades sostenibles: nuestro desafío, sintetiza claramente el concepto de comunidad sostenible definiéndola como "[...] aquella que construye su territorio buscando un equilibrio entre aspectos económicos, ambientales, sociales, institucionales y humanos con una perspectiva de equidad en el largo plazo" (p.2).

Para lograr la consolidación como comunidad sostenible se establecieron las siguientes cuatro pautas: el qué, el dónde, el quién y el cómo. Se resalta que, en el componente "quién", existen o confluyen diversos actores claves del territorio, donde se incluyen, no 
solo a sujetos de las comunidades o líderes comunitarios, sino también a otras instituciones públicas, fundaciones, empresas y organizaciones de la sociedad civil.

Bajo este argumento de Villar, la empresa privada ejerce y actúa como el "quién”, en el interior de una comunidad, haciendo una contribución al desarrollo sostenible desde la dinámica social, porque desarrolla proyectos personales de emprendimiento que, finalmente, se consolidan en un negocio propio (autoempleo), permitiendo la generación de un ingreso económico necesario para mejorar la calidad de vida.

Precisamente, Henrekson (2008) afirma que una alternativa para reconstruir el tejido social en las comunidades afectadas por el conflicto armado es a través del incentivo e incidencia en la consolidación de proyectos de emprendimiento. La empresa privada, a través de sus diversas iniciativas y mecanismos, coadyuva a la autosuficiencia de las comunidades desplazadas, en su desarrollo social y económico.

El documento Advancing the Sustainable Development Goals by Supporting Peace: How Business Can Contribute, publicado por Naciones Unidas (2015, p. 30), establece que las empresas, al tratar de facilitar la igualdad de acceso a puestos de trabajo y realizar una inversión en la formación y orientación, dan una contribución significativa a la paz bajo ese contexto.

Ejemplo de la manera como el sector empresarial privado contribuye a la paz en una comunidad han sido las capacitaciones realizadas a hombres y mujeres jóvenes como técnicos de gas natural, los talleres orientados a capacitar a las mujeres cabezas de hogar respecto de la iniciación de un negocio. El capacitar a mujeres desplazadas permite la igualdad de género y el empoderamiento económico, otorgándoles a estas personas la capacidad de un mejor bienestar, desarrollo humano, social y cumplimiento de las libertades y derechos que tienen como personas.

Un artículo del Journal of Business Ethics (2010), titulado Business and Peace: Sketching the Terrain, menciona que las empresas pueden fomentar la paz a través de mecanismos como el fomento del desarrollo económico, la promoción de puestos de trabajo y la estimulación de inversión local. Las investigaciones de Fort y Schipani (2004) hacen mención a que las empresas pueden contribuir a la paz en al menos cuatro maneras:

1. Fomento al desarrollo económico.

2. Ejercicio de la segunda vía diplomática.

3. Adopción de los principios de evaluación externos, tales como transparencia y apoyo a un sistema legal.

4. Al nutrir sentido de comunidad, dentro de la empresa como en las zonas en las que se encuentra.

Al confrontar los resultados obtenidos de la investigación, con los propuestos por Fort y Schipani, se reafirma la forma como el sector empresarial privado o público contribuyen al desarrollo social y a la paz, a través de la implementación y ejecución de 
proyectos, en comunidades que viven bajo una situación vulnerable de pobreza, desplazamiento forzoso y marginalidad, fomentando a su vez el desarrollo social y económico, como ha sido el caso de aquellos grupos de desplazados que habitan en los barrios el Talón y Bonanza.

Ralph y Conley Tyler (2006) proponen un modelo denominado Inter-Business Track Diplomacy (ITBD), que busca ayudar a las empresas a desarrollar su capacidad interna y participar como constructores de la paz en sus relaciones con los interesados a través de cuatro esferas de influencia: actividad principal, inversión social, promoción de políticas públicas junto con la creación de instituciones cívicas y construcción de la paz. Cabe resaltar que en la esfera Actividad principal, mencionan que las empresas u organizaciones deben promover la comprensión y tolerancia étnica en el lugar de trabajo, si es posible contratar a personas locales y fomentar la igualdad en la creación de riqueza, apoyando las oportunidades comerciales locales y proporcionando capacitación para la población local.

Por ello existe un gran compromiso de responsabilidad social empresarial por parte de las organizaciones públicas y privadas ante los impactos sociales, económicos, ambientales que ocasionan en la sociedad y en cada uno de aquellos que hacen parte de su grupo de interés. Esta relación recíproca frente a las comunidades lleva a que se busquen mecanismos que contribuyan al desarrollo sostenible y a la paz, satisfaciendo sus necesidades de una forma responsable, con el fin de no comprometer los recursos que llegaren a demandar las generaciones futuras y permitiéndoles a estas, como a las presentes, la generación de un progreso, desarrollo social, económico y ambiental.

Las diferentes prácticas adoptadas por las empresas se tornan en prácticas sostenibles, debido a que estas se convierten en acciones, actividades y métodos por los cuales una empresa contribuye socioambientalmente. Las organizaciones, sin importar su actividad y fin comercial, deben involucrar en su planeación estratégica, la forma de contribuir, de manera significativa y contundente, a mejorar la calidad de vida de la sociedad y mitigar el deterioro ambiental (Aristizábal, 2012).

Correa, Flynn y Amit (2004) afirman que las empresas u organizaciones deben tener un enfoque que contribuyan al desarrollo sostenible, al mejoramiento de la calidad de vida de la sociedad y de las personas en general.

Los Principios del Pacto Global, la estrategia Negocios para la Paz y los 17 Objetivos de la Agenda 2030 para el Desarrollo Sostenible son los que han establecido y promovido acciones para el desarrollo de nuestras sociedades, que convergen hacia la paz y el desarrollo sostenible, junto con el apoyo e influencia que ejercen las empresas en las comunidades. Acciones como promoción de derechos humanos, participación política, proyectos de desarrollo económico y social son medidas que refuerzan y consolidan la construcción de la paz (Prandi \& Lozano, 2010). Esto es reafirmado por la Agenda 2030 para el Desarrollo Sostenible, donde se acota: "No puede haber desarrollo sostenible sin paz, ni paz sin desarrollo sostenible". 


\section{Resultado de la investigación frente al desarrollo humano}

A lo largo del proceso investigativo y de acuerdo con los resultados obtenidos, donde se evidenciaron prácticas y acciones por parte una organización empresarial privada, que fortalecieron y forjaron el progreso en la vida de las personas que viven bajo condición de desplazamiento, se evidencia un vínculo con los postulados que propone Rey (2002) frente al desarrollo humano: obtener acceso a la educación, mejorar la calidad de vida y el fortalecimiento de las capacidades que tienen las personas llevan al progreso de la vida misma y bienestar humano. Por ello, el avance de esta investigación, a pesar de que su fin no era generar una teoría, sí permitió establecer ejemplos claros para que otras organizaciones empresariales contribuyan al desarrollo humano sostenible y a la paz en las comunidades donde operan.

Las organizaciones empresariales, dentro de sus lineamientos de responsabilidad social empresarial, aportan al desarrollo humano cuando estas buscan la manera para que las personas que viven en condiciones menos favorables logren obtener una mejor posibilidad de satisfacer sus necesidades fundamentales, bien sea a través de educación, salud y empleo, contribuyendo en su calidad de vida, donde todo ello se traduce en un aporte significativo al desarrollo de una comunidad, sociedad, pueblo y país.

\section{Resultados y relación con los objetivos planteados}

Los programas desarrollados por parte de un sector empresarial privado en el municipio de Turbaco han demostrado la manera como se puede contribuir al desarrollo sostenible y la paz gracias a los proyectos personales de emprendimiento y las capacitaciones realizadas a hombres y mujeres, para ser involucrados en un sector de la industria o permitiéndoles la autosuficiencia mediante un negocio propio.

Los diferentes resultados de la investigación permiten conocer que la población beneficiada por los programas implementados por la empresa privada ha reflejado una mejor calidad de vida y condición social, al permitírseles la inserción a una vida laboral. Lo anterior significó para algunos jóvenes desplazados una independencia económica de sus padres y el poder continuar con estudios de educación técnica. Al beneficiarse los desplazados mediante la formación impartida se forjan como mejores ciudadanos dentro de la comunidad, lo cual genera paz en la misma debido a que se rompe el ciclo de inseguridad y de pobreza.

Las técnicas utilizadas permiten identificar por parte de la comunidad desplazada nuevos mecanismos y planes encaminados al avance de la construcción de la paz y el desarrollo social mediante los programas educativos, capacitaciones a la juventud en temas de sexualidad y prevención de drogas, ya que estos contribuyen aún más al forjamiento de la paz en las comunidades desplazadas. Se identifica que la adhesión a la iniciativa Negocios por la Paz por parte de las empresas privadas es un mecanismo que facilita y ayuda en el avance y construcción de paz. De otra parte, los programas Brilla de Surtigas y Jóvenes con Valores Productivos se identifican como planes que dan la oportunidad a familias desplazadas de escasos recursos para mejorar sus condiciones de vida y la posibilidad de ser insertados laboralmente en la sociedad. Este último programa permite la 
equidad de género al capacitar a mujeres que demostraron tener las mismas o mejores capacidades para desempeñarse como técnicos en gas natural.

\section{A modo de conclusión}

Se considera que los programas que fueron extensivos a la población desplazada, por parte de una empresa privada, han contribuido a la paz porque favorecieron a un determinado grupo poblacional, mejorando su calidad de vida, su condición social y superación de la pobreza. Todo ello, mediante nuevas oportunidades de inserción laboral, aportando a su vez en la creación de un desarrollo equitativo en la comunidad.

Gracias a los talleres sobre la iniciación de negocios, que se realizaron en Cartagena y donde fueron partícipes madres desplazadas y cabezas de hogar que habitan en el municipio, se logró un aporte significativo al desarrollo social y económico en las vidas de ellas, ya que su condición socioeconómica les permitió en algunos casos lograr la iniciación de negocios propios, mejorando el nivel de vida y bienestar en su núcleo familiar.

El desarrollo social es percibido por jóvenes víctimas del desplazamiento mediante la generación de ingresos para el hogar, al momento de otorgárseles la oportunidad de ser partícipes en programas implementados como el de Jóvenes con Valores Productivos, lográndose en ellos la empleabilidad y una fuente de ingreso para el sustento de su hogar.

El sector empresarial privado, a través de las fundaciones sin ánimo de lucro, contribuye en el mejoramiento de la calidad de vida, el bienestar y desarrollo social en un sector poblacional que vive en condición de desplazamiento en el municipio de Turbaco, a través del desarrollo, ejecución e implementación de diferentes proyectos y programas de responsabilidad social empresarial.

La educación y formación que recibieron algunos de los que compartieron sus historias de vida es una muestra de la forma de disminuir la pobreza en una comunidad y a su vez establece lineamientos para minimizar la violencia e inseguridad que se pueda presentar en la región.

Se concluye que existen diversos factores que pueden impedir la adecuada ejecución de los programas propuestos por una empresa privada, encontrándose como los más influyentes los siguientes:

1. Falta de apoyo y compromiso por parte de entes municipales hacia programas propuestos por la organización. A pesar de que solo el $9 \%$ de los encuestados creen que este es un factor importante, es corroborado al existir una falta de iniciativa por parte de actores sociales e institucionales, siendo este un criterio de elegibilidad para que se lleven a cabo los programas en las comunidades desplazadas.

2. La falta de una caracterización y desactualización del registro de víctimas desplazadas por parte de la alcaldía muestran la falta de apoyo de los entes municipales hacia estas comunidades, que manifiestan su desmotivación y falta de credibilidad hacia los programas que en el futuro puedan ser implementados. 
Cuando las organizaciones empresariales del orden local, nacional e internacional deseen llevar a cabo programas bajo un enfoque de responsabilidad social en este tipo de comunidades y encuentren la existencia de algunos de los factores problemáticos mencionados, existen menos opciones para que se ejecuten e implementen programas que fomentan el desarrollo social y la paz en comunidades desplazadas.

Gracias a los resultados de esta investigación, se identificaron dos programas, Brilla y Jóvenes con Valores Productivos, que se convierten en modelo a seguir y ser adaptados por otras organizaciones empresariales en el orden nacional e internacional y que, finalmente, permitan aportar al desarrollo social, económico y a la paz en las comunidades en donde se deseen implementar, mejorando la condición socioeconómica y calidad de vida de las personas partícipes.

Se concluye que las industrias del sector empresarial privado, al adherirse a la iniciativa de Negocios para la Paz, facilitan los resultados en sus prácticas de responsabilidad corporativa y la construcción de paz en aquellos territorios que han sufrido las consecuencias del conflicto colombiano. La adhesión a esta iniciativa lleva a asumir un compromiso con la paz en Colombia y hacia las comunidades, buscando con ello la gestión de causas sociales y acciones de reparación, incluyendo en los proyectos sociales a personas que han sido desplazadas y sufrido violación de los derechos humanos, demostrando de esta manera cómo una organización empresarial puede convertirse en promotora de paz y ayudar al desarrollo social, como ocurrió en el municipio de Turbaco, gracias a la gestión realizada por una empresa privada de la Costa Caribe.

\section{Referencias}

Aristizábal, L. (2012) La responsabilidad social en las prácticas sostenibles de los almacenes de grandes superficie Exito de la ciudad de Manizales. Tesis de maestría. Universidad de Manizales.

Bifani, P. (1997). Medio ambiente y desarrollo. México: Universidad de Guadalajara.

Cardona et al. (2004). Encrucijadas de la seguridad en Europa y las Américas. Bogotá: Universidad del Rosario.

Correa, M., Flynn, S. \& Amit, A. (2004). Responsabilidad social corporativa en América Latina: una visión empresarial. Santiago de Chile: Naciones Unidas. Recuperado el 1 de agosto de 2016 de http://www.cepal.org/es/publicaciones/5621-responsabilidad-social-corporativa-en-america-latina-una-vision-empresarial

Fort, T. \& Schipani, C.. (2004). The Role of Business in Fostering Peaceful Societies. Cambridge: Cambridge University Press.

Fundación Surtigas. (2014). Informe de sostenibilidad 2013. Cartagena: Fundación Surtigas.

Fundación Surtigas. (2015). Informe de sostenibilidad 2014. Cartagena: Fundación Surtigas.

Fundación Surtigas. (2014). Guia orientadora sobre responsabilidad social empresarial aplicación de los principios de la RSE en Surtigas. Cartagena: Fundación Surtigas.

Fundación Surtigas. (2013). Documentación de la experiencia jóvenes con valores productivos. Cartagena: Fundación Surtigas. 
Henrekson, R. (2008). The Political Economy of Entrepreneurship: An Introduction. En M. Henreksonand \& R. Douham, The Political Economy of Entrepreneurship Vol. I y II (pp.11-31). UK: Edward Elgar.

Kurtenbach, S. \& Romero, M. (2005). Análisis del conflicto en Colombia. Bogotá. Recuperado el 10 de noviembre 2014 de: http://www.colombiassh.org/reh/IMG/ pdf_Causas _del_conlficto_armado_en_Colombia_charla_1_pag_1_a_40-_semana_2.pdf

Max-Neef, M., Elizalde, A. \& Hopenhayn, M. (1994). Desarrollo a escala humana: conceptos, aplicaciones y algunas reflexiones. Barcelona: Icaria Editorial.

Muñoz et al. (2005). Investigación de la paz y los derechos humanos desde Andalucía. Granada: Universidad de Granada. Recuperado el 27 de noviembre de 2014 de: http://www.ugr.es/ fmunoz/documentos/ pazddhhand.pdf

Oetzel, J., Westermann-Behaylo, M., Koerber, C., Fort, T., \& Rivera, J. (2010). "Business and Peace: Sketching the Terrain". Journal of Business Ethics, 89, 351-373

Organización de las Naciones Unidas. (1992). An Agenda for Peace: Preventive Diplomacy, Peacemaking and Peacekeeping. Recuperado el 18 de julio de 2015 en: http://www.un-documents.net/a47-277.htm.

Paz, N. (2017). Negocios para la paz. elespectador.com. Recuperado de: https://www. elespectador.com/noticias/economia/negocios-paz-articulo- 457365

Prandi, M. \& Lozano, J. (2010). La RSE en contextos de conflicto y postconflicto: de la gestión del riesgo a la creación de valor. Barcelona: Escola de Cultura de Pau (UAB)/ Instituto de Innovación Social (Esade). Recuperado el 15 junio 2016 de http://escolapau.uab.cat/img/programas/derecho/webRSE.pdf

Ralph, N. \& Conley, T. (2006). Companies as Peacebuilders: Engaging Communities Through Conflict Resolution. Australia: Universidad de Melbourne. Recuperado el 27 de julio de 2016 de: https://publications.qld.gov.au/storage/f/2014-0206T05\%3A57\%3A 27.9 78Z/ralph-natalie-final.pdf

Rettberg, A. (2003). Diseñar el futuro: una revisión de los dilemas de la construcción de paz para el postconflicto. Revista de Estudios Sociales, 15 (15-28). Universidad de Los Andes. Consultado el 30 de noviembre de 2014 en: http://res.uniandes.edu.co/view. php/471/index.php?id=47

Rey, G. (2002). Cultura y desarrollo humano: unas relaciones que se trasladan. Pensar Iberoamérica Revista de cultura. Consultado el 5 de marzo de 2015 en: http://www. oei.es/pensariberoamerica/ric00a04.htm

United Nations Global Compact. (2015). Advancing the Sustainable Development Goals by Supporting Peace: How Business Can Contribute. Recuperado el 15 de mayo de 2016 de: https://www.unglobalcompact.org/docs/issues_doc/Peace_and_Business/ Advanc ingSDGsPeace.pdf

Villar, R. (2015). Comunidades sostenibles: nuestro desafío. Bogotá: Red América. Recuperado el 10 de junio de 2016 de: http://www.redeamerica.org/ Portals/0/Publicaciones /DesarrolloBase/ComunidadessosteniblesESP. pdf?ver=2016-02-16-105536-473

Unesdoc.unesco.org. (2017). Disponible en: http://unesdoc.unesco.org/ images/0021/002167/216756s.pdf [Consultado 17 noviembre 2017]. 